\title{
Carrier-Induced Energy Shift in GaAs/AlGaAs Multiple Quantum Well Laser Diodes
}

\author{
P. A. Chen, Student Member, IEEE, C. Juang, Member, IEEE, and C. Y. Chang, Fellow, IEEE
}

\begin{abstract}
Emission energy shift due to high carrier density at threshold in multiple quantum well (MQW) laser diodes is investigated theoretically. This energy shift is evaluated through the Schrödinger and the Poisson equations self-consistently as well as the calculation of the gain spectra with carrier-dependent lifetime broadening. The band filling and the gain broadening effects show a blue shift on the emission energy. Larger number of wells, lower barrier height, or wider well thickness, reduces the blue shift dependence on the carrier density. At high injections, this blue shift is offset by the bandgap shrinkage effect, which displays smaller influence on MQW's. While the carrier density is further increased, the transition due to the second quantized state is found in single quantum wells, however it is difficult to be observed in MQW's.
\end{abstract}

\section{INTRODUCTION}

QEMICONDUCTOR quantum well (QW) structures Shave attracted numerous interests for their novel electronic and optical properties [1], [2]. Up to date, there have been promising characteristics in applications to many devices, such as modulation doped field effect transistors, photodetectors, QW lasers, photonic switchings, modulators, ...., etc. Recently, QW structures are extensively used in semiconductor lasers [4], [5], [8]. The QW lasers with staircase-like density of states are more favorable in laser actions than the conventional double heterostructure (DH) lasers with parabolic density of states. Some of the previous theoretical [7], [32], [33] and experimental [4], [5], [8] works have shown significant improvements on the static and dynamic behaviors of $\mathrm{QW}$ lasers.

During the operations of multiple quantum well (MQW) lasers, the interactions between carriers (e-e, h-h, and e-h) may cause the emission energy shift. This shift is important on the device designs and applications. The experiments show that the emission energy in MQW structures is somewhat smaller than its related absorption edge [9]. The red shift in the lasing wavelength has been first explained as LO-phonon participation by Holonyak et al. [4] because the lasing energy in optically pumped

Manuscript received April 20, 1992; revised November 30, 1992. This work was supported by the National Science Council, Republic of China under Project NSC 81-0417-E-009-12 and by the Optoelectronics and Systems Laboratories of ITRI, Republic of China, under Project 38N1200.

P. A. Chen and C. Y. Chang are with the Institute of Electronics, National Chiao-Tung University, Hsinchu 300, Taiwan, Republic of China.

C. Juang is with the Optoelectronics and Systems Laboratories (T200), ITRI, Hsinchu 310, Taiwan, Republic of China.

IEEE Log Number 9211547.
$\mathrm{GaAs} / \mathrm{Al}_{x} \mathrm{Ga}_{1-x} \mathrm{As}$ MQW structures lased at an energy $\hbar \omega \approx 36 \mathrm{meV}$ below the lowest confined electron to hole recombination energy $\left(E_{n=1, c \rightarrow h h}\right)$. However, in current injection MQW structures, Iwamura et al. [3] have observed that the results measured by comparing the spontaneous emission spectra and the stimulated emission spectra under various level injections were distinct from those described by the LO-phonon assisted recombination model. Tarucha et al. [10] have suggested the concept of carrier induced energy shift at high carrier injections, and have introduced modified bulk theory of many body effect by taking effective mass anisotropy into consideration. Nevertheless, the results by three dimensional treatment are not completely in accord on two dimensional characteristics. Tomita et al. [11] have considered two dimensional bandgap shrinkage effect to calculate energy shift dependence on carrier density in QW structures.

Due to quantum size effect, discrete energy levels in MQW structures are formed. Energy levels in an MQW structure can be tailored by changing the well number, well width, barrier height, barrier width, and shape of a well with different composition (such as GRIN-SCH, $\mathrm{SCH}$ structures), ..., etc. These factors affect the shift of emission energy induced by carriers. So it is an interesting topic to investigate carrier induced energy shift about MQW laser diodes in detail.

In this paper, the energy levels and their associated wavefunctions in MQW structures are calculated by solving both the Schrödinger and the Poisson equations selfconsistently. The linear gain coefficient based on the standard perturbation theory (Fermi's Golden Rule) is calculated. In addition, the gain broadening mechanism resulting from intraband scattering is also considered. Fig. 1 illustrates the MQW structure to be discussed. The free carrier screening effect is taken into consideration, and the bandgap shrinkage effect solved by the local density functional method [12] is also incorporated in the calculation of MQW energy states. All of the energy states are obtained under steady state operations, and those are used to calculate the gain spectra and the lasing wavelength shift in MQW laser diodes. To our knowledge, though there exist a number of models [13]-[15], [17], [22], [25], [29], [33] to solve MQW laser's problems, little work has been reported to apply a self-consistent manner with consideration of the bandgap shrinkage effect to treat MQW lasers systematically. 


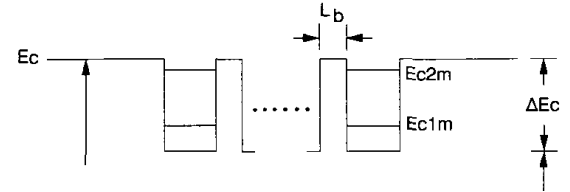

$\mathrm{Eg}\left(\mathrm{Al}_{\times} \mathrm{Ga}_{1-\mathrm{x}} \mathrm{As}\right)$

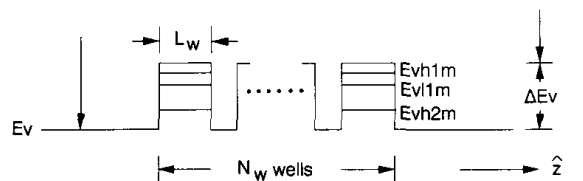

Fig. 1. Schematic potential profiles of MQW structures. A series of bound states $E_{c n m}\left(n=1,2,3, \cdots, m=1,2, \cdots, N_{w}\right), N_{w}$ is the well number, for electrons exist in the conduction band, $E_{r h n m}$ for heavy holes, and $E_{v / r m}$ for light holes exist in the valence band.

The organization of this paper is as follows. In Section II, numerical techniques for solving the time-independent Schrödinger equation and the one dimensional Poisson equation are briefly discussed. The self-consistent method is used to link these two equations. Using the perturbation theory (Fermi's Golden Rule), the MQW gain function with carrier density-dependent lifetime broadening is calculated. In Section III, the emission energy shifts via the band filling and the gain broadening effects according to different injections are compared and analyzed for related parameters of MQW structures, i.e., well number, barrier height, well width, and barrier width. Finally, comparisons about band structures, emission energy shifts, and gain spectra with or without the bandgap shrinkage effect are given in Section IV.

\section{Numerical Techniques}

\section{A. Time-Independent Schrödinger Equation}

A simple method has been developed for obtaining the solutions of the time-independent Schrödinger equation with arbitrary finite potential energy. The algorithmic steps have been thoroughly discussed by Juang et al. [16]. In this work, the technique is expanded to solve the MQW structures. The modified method can get all of the energy levels and their associated wavefunctions. The important concepts about this method are distinctly described below.

Within the framework of effective mass approximation, the one dimensional time-independent Schrödinger equation is

$$
H \Psi(z)=E \Psi(z),
$$

where $\Psi(z)$ is the envelope wavefunction, and $\hat{z}$-direction is perpendicular to the well. $H$ is the Hamiltonian made of BenDaniel and Duke's effective Hamiltonian

$$
H=\frac{-\hbar^{2}}{2} \frac{\partial}{\partial z}\left(\frac{1}{m^{*}(z)} \frac{\partial}{\partial z}\right)+V(z),
$$

where $m^{*}(z)$ is the position-dependent effective mass.
According to finite difference method, (1) can be discretized as

$$
\begin{aligned}
& \frac{-\hbar^{2}}{\Delta z_{j}^{2}}\left(\frac{\Psi_{j+1}}{m_{j+1}^{*}+m_{j}^{*}}+\frac{\Psi_{j-1}}{m_{j-1}^{*}+m_{j}^{*}}-\frac{\Psi_{j}}{m_{j+1}^{*}+m_{j}^{*}}\right. \\
& \left.-\frac{\Psi_{j}}{m_{j-1}^{*}+m_{j}^{*}}\right)+V_{j} \Psi_{j}=E \Psi_{j},
\end{aligned}
$$

where $\Delta z_{j}$ is the discretization space under uniform grid distribution and is set as $1 \AA$ in this calculation.

This difference equation can be written in matrix form $\hat{H} \Psi=E \Psi$. Multiplying the starting vector $\Psi^{(0)}$ by $(\hat{H}-$ $\mu I)^{-K}$, and we can obtain

$$
\begin{aligned}
& (\hat{H}-\mu I)^{-K} \cdot \Psi^{(0)} \\
& \quad=\frac{a_{1} \Psi_{1}}{\left(E_{1}-\mu\right)^{K}}+\frac{a_{2} \Psi_{2}}{\left(E_{2}-\mu\right)^{K}}+\cdots+\frac{a_{n} \Psi_{n}}{\left(E_{n}-\mu\right)^{K}},
\end{aligned}
$$

where $\mu$ is an adjustable parameter, $\Psi_{1}, \Psi_{2}, \cdots, \Psi_{n}$ are the eigenvectors, and $E_{1}, E_{2}, \cdots, E_{n}$ are the eigenvalues of the matrix $\hat{H}$. If $\mu$ is much closer to $E_{1}$ than to any other eigenvalues, $(\hat{H}-\mu I)^{-K} \cdot \Psi^{(0)}$ will have a dominant component in the direction of $\Psi_{1}$ and $E_{1}$, then the solution is retrieved while the iteration number $K$ becomes large. This technique is often referred to as the inverse power method.

Much computing time reduction is attained by carefully selecting the starting vector $\Psi^{(0)}$ and the initial guess $\mu$. In this work, $\Psi^{(0)}$ is given as a linear combination of both symmetry and anti-symmetry functions with reference to the center point of the structure. This initialization vector increases the convergence rate significantly. In the beginning, the initial guess $\mu$ is set to be the value at the lowest bottom (top) of conduction (valence) band, and is gradually increased (decreased) to get the corresponding eigenvalues until the lowest top (bottom) of conduction (valence) band. Because of the coupling effect in MQW, the scanned interval in finding splitting subbands (e.g., $E_{c 1 m}$, $m=2, \cdots, N_{w}$, the splitting subbands of ground state) is changed smaller than that in finding the next energy level (e.g., $E_{c n}, n=2,3, \cdots$, etc.: the first splitting subband of the first excited state, second excited state, ... , etc.). However, any interval must be ensured smaller than the spacing of the nearest two levels.

\section{B. One Dimensional Poisson Equation}

The one dimensional Poisson equation taking position dependence of dielectric constant into consideration is expressed as

$$
\begin{aligned}
& \frac{\partial}{\partial z}\left[\epsilon(z) \frac{\partial}{\partial z} \phi(z)\right] \\
& \quad=-q\left[N_{D}^{+}(z)-N_{A}^{-}(z)+p(z)-n(z)\right],
\end{aligned}
$$

where $q$ represents elementary charge equal to $1.6 \times$ $10^{-19}$ coulomb, $\epsilon(z)$ is the position-dependent dielectric constant in each layer material, and $\phi(z)$ is the electro- 
static potential. For simplicity, the MQW structures are considered to be undoped. Both ionized donor concentration $N_{D}^{+}(z)$ and ionized acceptor concentration $N_{A}^{-}(z)$ are set to be zero. Hence, only the injected electrons concentration $n(z)$ and holes concentration $p(z)$ distributions are taken into account. The Poisson equation can be solved by the same way discussed in the previous part, thus (5) is transformed into its difference form

$$
\begin{aligned}
& \frac{1}{2\left(\Delta z_{i}\right)^{2}}\left[\left(\epsilon_{i+1}+\epsilon_{i}\right) \phi_{i+1}+\left(\epsilon_{i}+\epsilon_{i-1}\right) \phi_{i-1}\right. \\
& \left.-\left(\epsilon_{i+1}+\epsilon_{i-1}+2 \epsilon_{i}\right) \phi_{i}\right]=-q\left(p_{i}-n_{i}\right),
\end{aligned}
$$

where $\Delta z_{i}$ is uniformly partitioned, to be $1 \AA$. Since there is no external voltage applied, both end side potentials are fixed at zero as the boundary conditions, i.e., $\phi(-N t / 2)$ $=0 \mathrm{~V}, \phi(N t / 2)=0 \mathrm{~V}$, where the total grid number is $N t+1$ ( $N t$ is an even number). Equation (6) can be expanded into a tridiagonal matrix style $\hat{A} x=b$. Using Gaussian elimination and back-substitutional method, the solution $\phi(z)$ can be easily obtained.

\section{Self-Consistent Calculation with Screening Effect and Bandgap Shrinkage Effect}

In the MQW structures, the Schrödinger and the Poisson equations are connected together by the self-consistent method [18], where the free carrier screening potential is induced by the charged carriers, and inherently included in the calculations. Moreover, both conduction band and valence band are assumed to be parabolic bands, and mixing effects between heavy hole and light hole are neglected. Sugimura [17] has considered the effect of Lvalley transition in determining the threshold current density. So at high carrier injections, the non-radiative part due to L-valley transition is calculated, and contributes to the $\Gamma$-valley in the well region.

The Hamiltonian for this system can be written as

$$
H=H_{\mathrm{e}}+H_{\mathrm{hh}}+H_{\mathrm{lh}},
$$

and

$$
\begin{aligned}
H_{i}= & \frac{-\hbar^{2}}{2}\left(\frac{\partial}{\partial z} \frac{1}{m_{i \perp}^{*}(z)} \frac{\partial}{\partial z}\right) \\
& +V_{i}(z), \quad i=\mathrm{e}, \mathrm{hh}, \mathrm{lh},
\end{aligned}
$$

where $H_{\mathrm{e}}\left(H_{\mathrm{hh}}, H_{\mathrm{lh}}\right)$ describe the motions of electrons (heavy holes, light holes) along $\hat{z}$-direction in MQW structures. The solutions of wavefunctions are defined as $\Psi(z)=\Psi_{\mathrm{e}}(z) \Psi_{\mathrm{hh}}(z) \Psi_{1 \mathrm{~h}}(z)$, where $\Psi_{\mathrm{e}}(z), \Psi_{\mathrm{hh}}(z)$, and $\Psi_{\mathrm{lh}}(z)$ represent the envelope functions of electrons, heavy holes, and light holes respectively. $m_{i \perp}^{*}(z)$ is the position dependent effective mass perpendicular to the interface. The effective potential energy $V_{i}, i=\mathrm{e}, \mathrm{hh}$, lh is given by

$$
V_{i}(z)=-q \phi_{i}(z)+\Delta E_{i}(z)
$$

where $\phi_{i}(z)$ is the electrostatic potential, and $\Delta E_{i}(z)$ is the effective potential energy associated with the heterojunction discontinuity (assume square well in the calculations, and the band discontinuities for conduction and valence bands are shown as $\left.\Delta E_{c}=0.6 \Delta E_{g}, \Delta E_{v}=0.4 \Delta E_{g}[23]\right)$.

While the bandgap shrinkage effect is included, the effective potential energy is represented by

$$
V_{i}(z)=-q \phi_{i}(z)+\Delta E_{i}(z)+V_{x c i}(z),
$$

where $V_{x c i}(z)$ is the local exchange-correlation potential energy due to electron-electron or hole-hole interactions. At high carrier density, the bandgap narrowing mostly results from the exchange-correlation potential. Taking the local density functional approximation (LDA) [12], the value of $V_{x c i}(z)$ can be described by a simple analytic parametrization [18]:

$$
\begin{aligned}
V_{x c i}(z)= & -\left[1+0.07734 x_{i} \ln \left(1+x_{i}^{-1}\right)\right] \\
& \cdot\left(\frac{2}{\pi_{\alpha i} r_{s i}}\right) R_{y i}^{*},
\end{aligned}
$$

where

$$
\begin{aligned}
& \alpha_{i}=\frac{4}{(9 \pi)^{1 / 3}}, \\
& x_{i}=x_{i}(z)=\frac{r_{s i}}{21}, \\
& r_{s i}=r_{s i}(z)=\left[\frac{4}{3} \pi a_{i}^{* 3} n_{i}(z)\right]^{1 / 3}, \\
& a_{i}^{*}=\frac{4 \pi \epsilon_{i}(z) \hbar^{2}}{m_{i}(z) q^{2}}, \\
& R_{y i}^{*}=\frac{q^{2}}{8 \pi \epsilon_{i}(z) a_{i}^{*}},
\end{aligned}
$$

$m_{i}(z)$ is the local effective mass, and $R_{y i}^{*}$ is the Rydberg number. The exchange-correlation potential energy $V_{x c i}(z)$ strongly depends on the carrier density $n_{i}(z)$. When compared with the screening effect, the exchange-correlation potential energy $V_{x c i}(z)$ influences more on the potential profile than the screening effect [11], [19].

The electrostatic potential $\phi(z)$ is obtained from the Poisson equation under the condition of $N=P$ for charge neutrality, $N, P$ mean the injected carrier densities for electrons and holes respectively. The representation of the Poisson equation is

$$
\frac{\partial}{\partial z}\left[\epsilon(z) \frac{\partial \phi(z)}{\partial z}\right]=-q[p(z)-n(z)] .
$$

The charge densities $p(z)$ and $n(z)$ about holes and elec- 
trons are given by [17], [19]

$$
\begin{aligned}
p(z)= & \frac{\bar{m}_{\mathrm{hh}}^{*} \kappa_{B} T}{\pi \hbar^{2}} \sum_{j}\left|\psi_{\mathrm{hhj}}(z)\right|^{2} \ln \left[1+\exp \left(\frac{E_{\mathrm{hhj}}-E_{f p}}{\kappa_{B} T}\right)\right] \\
& +\frac{\bar{m}_{\mathrm{lh}}^{*} \kappa_{B} T}{\pi \hbar^{2}} \sum_{j}\left|\psi_{\mathrm{lhj}}(z)\right|^{2} \ln \left[1+\exp \left(\frac{E_{\mathrm{lhj}}-E_{f p}}{\kappa_{B} T}\right)\right], \\
n(z)= & \frac{M_{\Gamma} \bar{m}_{\Gamma \mathrm{e}}^{*} \kappa_{B} T}{\pi \hbar^{2}} \sum_{j}\left|\psi_{\Gamma \mathrm{ej}}(z)\right|^{2} \ln \left[1+\exp \left(\frac{E_{f n}-E_{\Gamma \mathrm{ej}}}{\kappa_{B} T}\right)\right] \\
& +\frac{M_{L} \bar{m}_{L e}^{*} \kappa_{B} T}{\pi \hbar^{2}} \sum_{j}\left|\psi_{L e j}(z)\right|^{2} \ln \left[1+\exp \left(\frac{E_{f n}-\Delta E_{\Gamma L}-E_{l e j}}{\kappa_{B} T}\right)\right],
\end{aligned}
$$

and

$$
\begin{aligned}
& P=\int_{-\infty}^{\infty} \frac{p(z) d z}{L_{w k j}}, \\
& N=\int_{-\infty}^{\infty} \frac{n(z) d z}{L_{w k j}},
\end{aligned}
$$

where $M_{\Gamma}, M_{L}$ are the number of equivalent minima in the $\Gamma$-valley and L-valley respectively $\left(M_{\Gamma}=1, M_{L}=4\right.$ for GaAs, $\Delta E_{\Gamma \mathrm{L}}$ is the energy difference between $\Gamma$-valley and L-valley, $E_{f n}, E_{f p}$ mean the quasi-Fermi levels for electrons and holes, and

$1 / \bar{m}_{i}^{*}=\int_{-\infty}^{\infty}\left|\psi_{i}(z)\right|^{2} d z / m_{i}^{*}(z), \quad(i=\Gamma \mathrm{e}, \mathrm{Le}, \mathrm{hh}, \mathrm{lh})$.

Moreover, $L_{w k j}\left(k=1,2, \cdots, N_{w}\right)$ represent the well width of the $k$ th well number about the $j$ th subband in an MQW structure. Assume all of well widths are the same size in the calculations, i.e., $L_{w 1 j}=L_{w 2 j}=L_{w 3 j}=\cdots$ $=L_{w}$.

According to (7)-(12), the Schrödinger and the Poisson equations are solved self-consistently. The flow chart is shown in Fig. 2.

During iteration process, the "fixed convergence factor" method proposed by Stern [20] is used to determine the next input potential,

$$
V^{n+1}(z)=V^{n-1}(z)+\Gamma\left(V^{n}(z)-V^{n-1}(z)\right),
$$

where $\Gamma$ is a fixed convergence factor, and $0<\Gamma \leq 1$. Determination of $\Gamma$ significantly affects convergence rate. Larger $\Gamma$ reduces iteration times, but may cause divergence. Small $\Gamma$ assures convergence, however, the computing time becomes longer. There exists a trade-off between convergence speed and computing time. In this work, the better choice of convergence factor $\Gamma$ is about 0.8 for a single QW $\left(L_{w}=101 \AA, x_{\mathrm{Al}}=0.3\right.$, and $N_{\text {th }}=$ $5 \times 10^{18} \mathrm{~cm}^{-3}$, without the bandgap shrinkage effect), and decreases to a lower value as the well number increases. Fig. 3 shows different convergence rates at variant $\Gamma$ values for a double quantum well, and a better choice of $\Gamma$ is around 0.6 .

\section{D. $M Q W$ Gain Function}

The calculation of optical gain is based on the standard perturbation theory (Fermi's Golden Rule). The expression for the MQW gain coefficient with carrier densitydependent lifetime broadening included may be written as [25], [29]

$$
\begin{aligned}
g(E)= & \frac{\omega}{n_{e q}} \mu_{0} c_{0} \sum_{\substack{i=\mathrm{e} \\
j=\mathrm{hh}, \mathrm{lh}}} \int_{E_{g}+E_{c i}+E_{v j}}^{\infty} \\
& \cdot\left|\left\langle\psi_{i} \mid \psi_{j}\right\rangle\right|^{2}\left|\hat{\xi} \cdot \hat{M}_{i j}\right|^{2} \\
& \cdot \rho_{\text {red }}^{i j}\left(E^{\prime}\right)\left(f_{c}\left(E^{\prime}\right)-f_{v}\left(E^{\prime}\right)\right) \\
& \cdot \frac{\left(\hbar / \tau_{\text {in }}\right)}{\left(E^{\prime}-E\right)^{2}+\left(\hbar / \tau_{\text {in }}\right)^{2}} d E^{\prime}
\end{aligned}
$$

where

$$
\begin{aligned}
& \omega: \quad \text { angular frequency of photons, } \\
& \mu_{0}: \quad \text { permeability, } \\
& c_{0}: \quad \text { speed of light in free space, } \\
& n_{\text {eq }} \text { : equivalent effective refractive index, } \\
& i, j \text { : conduction (e) and valence (hh, lh) } \\
& \text { bands quantum numbers, } \\
& \left|\left\langle\psi_{i} \mid \psi_{j}\right\rangle\right|^{2} \text { : the spatial overlap factor between } \\
& \text { states } i \text { and } j \text {, } \\
& \xi: \quad \text { polarization vector, } \\
& \hat{M}_{i j}: \quad \text { optical dipole momentum matrix ele- } \\
& \text { ment between states } i \text { and } j \text {, } \\
& \rho_{\text {red }}^{i j}: \quad \text { the reduced density of states, } \\
& f_{c}\left(E^{\prime}\right), f_{v}\left(E^{\prime}\right) \text { : Fermi-Dirac distribution functions for }
\end{aligned}
$$

Notice that no $k$-selection rule is followed due to intraband relaxation [34], and the $i=j(\Delta n=0)$ selection rule is also no longer imposed [32], i.e., all symmetryallowed transitions are considered. 


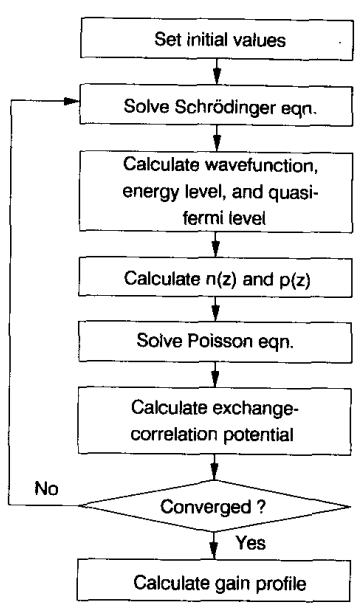

Fig. 2. Flow chart of self-consistent calculation by solving the Schrödinger and the Poisson equation.

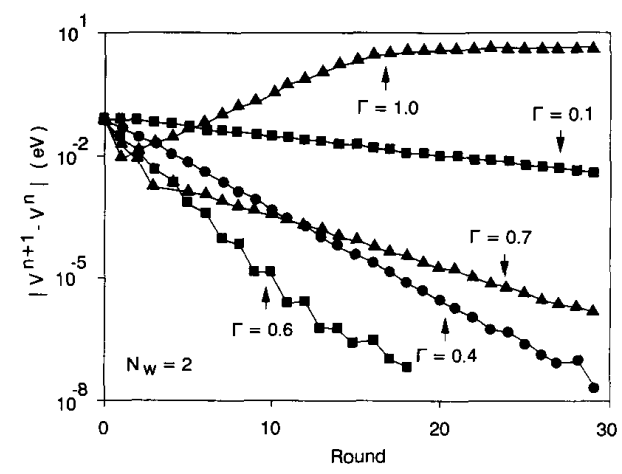

Fig. 3. Comparisons of convergence rates at $\Gamma=0.1,0.4,0.6,0.7,1.0$ for $N_{w}=2, L_{w}=101 \AA, L_{b}=51 \AA$, and $N_{\mathrm{th}}=5 \times 10^{18} \mathrm{~cm}^{-3}$, where $V^{n}, V^{n+1}$ mean the potential profiles after the $n$th and $(n+1)$ th iterations.

The modal gain $g_{\text {mod }}(E)$, which considers the confinement factor $\Gamma_{c}$ in the waveguide region, is given by [7]

$$
g_{\text {mod }}(E)=\Gamma_{c} g(E) \text {. }
$$

One of the main differences between the single $\mathrm{QW}$ and MQW lasers is that the confinement factor $\Gamma_{c}$ of the former is significantly smaller than that of the latter. Modal confinement factor $\Gamma_{c}$ in an MQW structure can be expressed by a simple formula [27]

$$
\begin{aligned}
\Gamma_{c}(\mathrm{MQW}) & =\frac{N_{w} L_{w}}{N_{w} L_{w}+\left(N_{w}-1\right) L_{b}} \gamma \\
\gamma & =\frac{\int_{d / 2}^{d / 2} F^{2}(x) d x}{\int_{-\infty}^{\infty} F^{2}(x) d x} \approx 2 \pi^{2}\left(\bar{n}_{1}^{2}-\bar{n}_{2}^{2}\right) d^{2} / \lambda_{0}^{2}
\end{aligned}
$$

where $F(x)$ is the electric field intensity in the waveguide of MQW structures, $\bar{n}_{1}^{2}$ is the equivalent refractive index in the active region, $\bar{n}_{2}^{2}$ is the equivalent refractive index outside the active region (i.e., in the cladding layer), $d$ is the active region thickness $\left(d=N_{w} L_{w}+\left(N_{w}-1\right) L_{b}\right.$ in this case), and $\lambda_{0}$ is the free space wavelength $\left(\lambda_{0} \approx\right.$ $8700 \AA$ in GaAs).

The equivalent effective refractive index $n_{\mathrm{eq}}$ of an MQW structure is computed via [15]

$$
n_{\mathrm{eq}}=\frac{N_{w} L_{w} n_{\text {well }}+\left(N_{w}-1\right) L_{b} n_{\text {barrier }}}{N_{w} L_{w}+\left(N_{w}-1\right) L_{b}},
$$

where $L_{b}$ is the barrier width, $n_{\text {well }}$ is the refractive index of well layer, $n_{\text {barrier }}$ is the refractive index of barrier layer in the active region.

The intraband relaxation time $\tau_{\text {in }}$ is considered as a carrier density-dependent lifetime, rather than using a constant lifetime of $1 \times 10^{-13} \mathrm{~s}$ [25], or using an energydependent lifetime [13], [25]. According to different injected carrier densities, the lifetime can be represented by [26]

$$
\tau_{\text {in }}=c \times 10^{-13}\left(\frac{N}{10^{18}}\right)^{-1 / 2},
$$

with the injected carrier density $N$ in $\mathrm{cm}^{-3}$, the intraband lifetime $\tau_{\text {in }}$ in $\mathrm{s}$, and $c$ is an adjustable parameter. This expression (with $c$ selected to be 2) has been successfully applied to gain profile calculation in GaAs/AlGaAs QW laser with $25 \AA$ wells [26].

The dispersion in the $k_{x}-k_{y}$ plane is taken to be parabolic and isotropic in the conduction and valence bands, therefore, the reduced density of state per unit volume in an MQW structure between $i$ th and $j$ th states is given by [29]

$$
\begin{aligned}
& \rho_{\text {red }}^{i j}=\frac{m_{r}^{i j}}{\pi \hbar^{2} L_{w} N_{w}}, \\
& \frac{1}{m_{r}^{i j}}=\frac{1}{m_{c i}}+\frac{1}{m_{v j}},
\end{aligned}
$$

where $m_{r}^{i j}$ is the reduced effective mass, and $m_{c i}, m_{v \mathrm{j}}$ denote the effective electron mass in the $i$ th band and the effective hole mass in the $j$ th band respectively.

The Fermi-Dirac distribution functions for electron occupancy probabilities in the conduction and valence bands are described as [13]

$$
\begin{aligned}
& f_{c}\left(E^{\prime}\right)=\frac{1}{1+e^{\left(E_{p c}-E_{f c}\right) / \kappa_{B} T}}, \\
& f_{v}\left(E^{\prime}\right)=\frac{1}{1+e^{-\left(E_{p^{\prime}}-E_{f v}\right) / \kappa_{B} T}},
\end{aligned}
$$

where

$$
\begin{aligned}
& E_{p c}=E_{c i}+\left(\frac{m_{r}^{i j}}{m_{c i}}\right)\left(E^{\prime}-E_{g}^{\mathrm{eff}}-E_{c i}-E_{v j}\right), \\
& E_{p v}=E_{v j}+\left(\frac{m_{r}^{i j}}{m_{v j}}\right)\left(E^{\prime}-E_{g}^{\mathrm{eff}}-E_{c i}-E_{v j}\right),
\end{aligned}
$$


$E_{g}^{\text {eff }}$ means the effective energy gap in the well layer. The relative energy origin for $E_{p c}, E_{f c}$, and $E_{c i}$ is at the bottom of the conduction band potential. The relative energy origin for $E_{p v}, E_{f v}$, and $E_{v j}$ is at the top of the valence band potential, and the positive direction is defined downward the potential profile.

The spatial optical momentum matrix elements are evaluated using the so called $k \cdot p$ method with four-band approximation developed by Kane [28]. The momentum matrix elements for TE modes transitions $(\hat{\xi} \perp \hat{z})$, are considered as below: [25], [30]

$$
\begin{aligned}
&\left|\hat{\xi} \cdot \hat{M}_{i j}\right|^{2}= \frac{3}{4}\left|M_{0}\right|^{2}\left(1+\cos ^{2} \theta_{i}\right), \\
&(\mathrm{e}-\text { hh transitions }), \\
&=\frac{1}{4}\left|M_{0}\right|^{2}\left(5-3 \cos ^{2} \theta_{i}\right), \\
&(\mathrm{e}-\text { lh transitions }) .
\end{aligned}
$$

The angular factor $\theta_{i}$ is the angle of $\hat{k}$-vector with respect to the $\hat{z}$-axis for the $i$ th conduction subband, [29]

$$
\cos ^{2} \theta_{i}=\frac{E_{c i}}{E_{c i}+E_{c i, x y}},
$$

where $E_{c i, x y}$ followed by a parabolic band energy means the adding energy in the $\hat{x}$ and $\hat{y}$ directions. $M_{0}$ is the bulk averaged momentum matrix element between conduction $\langle S|$ state and valence $|P\rangle$ state given by [28]

$$
\left|M_{0}\right|^{2}=\frac{e^{2} \hbar^{2}}{6 m_{c}^{*} E^{\prime 2}} \frac{E_{g}\left(E_{g}+\Delta\right)}{E_{g}+(2 / 3) \Delta},
$$

where $\Delta$ denotes the split-off valence band separation ( $\Delta \approx 0.34 \mathrm{eV}$ in GaAs), and $m_{c}^{*}$ means the conduction band relative effective mass.

\section{EMISSION ENERgy OF MQW LASER Diodes}

Emission energy due to high carrier injections at threshold is performed in $\mathrm{GaAs} / \mathrm{Al}_{x} \mathrm{Ga}_{1-x} \mathrm{As} \mathrm{MQW}$ laser diodes at room temperature $\left(300^{\circ} \mathrm{K}\right)$. The material parameters about $\mathrm{GaAs}$ and $\mathrm{Al}_{x} \mathrm{Ga}_{1-x}$ As system used in the calculations are shown below, and a brief summary for GaAs material is listed in the Table I.

The bandgap of $\mathrm{Al}_{x} \mathrm{Ga}_{1-x}$ As depending on the mole fraction of $\mathrm{Al}$ and operational temperature, is taken to be [21]

$$
\begin{aligned}
E_{g}= & 1.519+1.247 x-\frac{5.405 \times 10^{-4} T^{2}}{T+204} \\
& \text { if } x \leq 0.45, \\
= & 1.519+1.247 x-1.147(x-0.45)^{2} \\
& -\frac{5.405 \times 10^{-4} T^{2}}{T+204} \quad \text { if } x>0.45 .
\end{aligned}
$$

Moreover, the effective masses for electrons in the $\Gamma$-valley, for heavy holes, and light holes in the valence band
TABLE I

A Summary of GaAs Material Parameters used in the Calculations FOR GaAs $/ \mathrm{Al}_{x} \mathrm{Ga}_{1-x}$ As Heterostructure

\begin{tabular}{ll}
$m_{0}$ & $9.1 \times 10^{-28} \mathrm{~g}$ \\
$m_{\Gamma}$ & $0.067 m_{0}$ \\
$m_{\mathrm{L}}$ & $0.056 m_{0}$ \\
$m_{\mathrm{hh}}$ & $0.34 m_{0}$ \\
$m_{\mathrm{lh}}$ & $0.094 m_{0}$ \\
$T$ & $300^{\circ} \mathrm{K}$ \\
$\Delta E_{\Gamma \mathrm{L}}$ & $0.284 \mathrm{eV}$ \\
$E_{g}$ & $1.422 \mathrm{eV}$ \\
$\Delta$ & $0.34 \mathrm{eV}$ \\
$\lambda_{0}$ & $8700 \AA$ \\
$\epsilon_{0}$ & $8.854 \times 10^{-14} \mathrm{~F} / \mathrm{cm}$ \\
$\epsilon$ & $13.1 \epsilon_{0}$ \\
$\mu_{0}$ & $1.2566 \times 10^{-8} \mathrm{H} / \mathrm{cm}$ \\
$n_{r}$ & 3.59 \\
$\Delta E_{c} / \Delta E_{v}$ & $60 / 40$ \\
\hline
\end{tabular}

are demonstrated as [13]

$$
\begin{aligned}
& m_{\Gamma}(z) / m_{0}=0.067+0.083 x, \\
& m_{\mathrm{hh}}(z) / m_{0}=0.34+0.175 x, \\
& m_{\mathrm{lh}}(z) / m_{0}=0.094+0.069 x,
\end{aligned}
$$

where $m_{0}$ means the electron rest mass. The electron effective mass $m_{\mathrm{L}}$ in the L-valley is given by $m_{\mathrm{L}}(z) / m_{0}=$ $0.56+0.1 x$. The dielectric constant of $\mathrm{Al}_{x} \mathrm{Ga}_{1-x}$ As is represented by

$$
\epsilon(z)=(13.1-3 x) \epsilon_{0},
$$

where $\epsilon_{0}$ is the dielectric constant in the vacuum. The expression for the composition dependent refractive index is given by [13]

$$
n_{r}(x)=3.59-0.71 x+0.091 x^{2} .
$$

These parameters described above for GaAs and $\mathrm{Al}_{x} \mathrm{Ga}_{1-x} \mathrm{As}$ are used in the calculations.

In this Section, the bandgap shrinkage effect is neglected (discussed in the next Section), and only the free carrier screening effect is considered in calculating the energy levels and their associated wavefunctions. Furthermore, the emission energy is determined by the lasing wavelength of maximum modal gain peak for TE modes radiation in MQW lasers. Emission energy due to carrier density at threshold $\left(N_{\mathrm{th}}\right)$ are discussed in accordance with the effects of well number $\left(N_{w}\right)$, well width $\left(L_{w}\right)$, barrier height $\left(x_{\mathrm{Al}}\right)$, and barrier width $\left(L_{b}\right)$. Energy shift via the band filling and the gain broadening effects can be tailored by the MQW structure parameters. These physical effects are also discussed as follows.

\section{A. Effects of $Q W$ Number}

QW number is the most important design parameter of MQW lasers. Some characteristics of MQW lasers (such as threshold current density [14], or differential gain [7], . . , etc.) are determined by the number of wells. Fig. 4 shows comparisons of emission energy among single, double, and triple $\left(N_{w}=1,2,3\right) \mathrm{QW}$ lasers at high threshold carrier density $\left(N_{\mathrm{th}}=1 \times 10^{18}-1 \times 10^{19} \mathrm{~cm}^{-3}\right)$. 


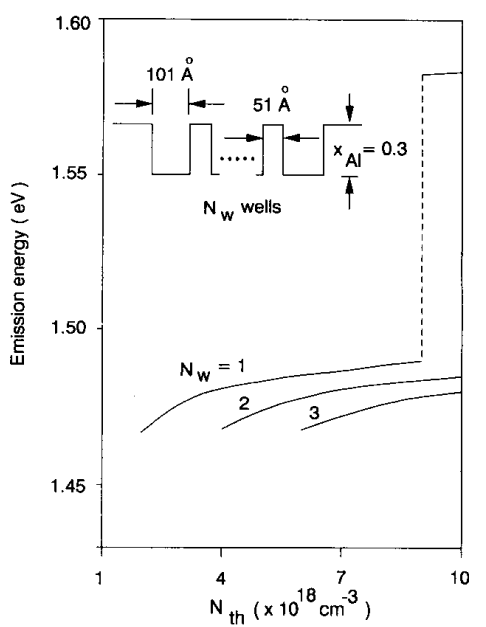

Fig. 4. Emission energy under high carrier injections $\left(N_{\text {th }}=1 \times 10^{18}-1\right.$ $\times 10^{19} \mathrm{~cm}^{-3}$ ) for various well number $N_{\mathrm{w}}=1,2,3$ and $L_{b}=51 \AA, L_{w^{\prime}}$ $=101 \AA, x_{\mathrm{Al}}=0.3$ (where the bandgap shrinkage effect is not considered).

The other parameters about barrier height, well width, and barrier width are $0.3,101$, and $51 \AA$ respectively.

As Fig. 4 shows, the lasing wavelength becomes short while the threshold carrier density increases. This blue shift is mostly decided by the band filling and the gain broadening effects (discussed in Section IV). The quasiFermi energy is increased to a higher side with an increase of carrier density, such that the band filling effect comes to be more obvious. In addition, the gain broadening effect also causes a blue shift at high injections. Thus the emission energy becomes larger while the carrier density is increased.

While the number of wells is increased, the modal gain peak is shifted to a longer wavelength. Moreover, the energy shift due to carrier density is less sensitive for a larger number of wells, and can be characterized by the quantity of $\Delta E / \Delta N_{\text {th }}\left(\Delta N_{\text {th }}=N_{\text {th }}-N_{\text {th } 0}\right)$, where $\Delta E$ denotes the emission energy shift from $N_{\text {tho }}$ to $N_{\text {th }}$, where $N_{\text {tho }}$ is defined as the minimum threshold carrier density at which the positive modal gain occurs $\left(N_{\text {th } 0} \approx 2 \times 10^{18}, 4 \times\right.$ $10^{18}$, and $6 \times 10^{18} \mathrm{~cm}^{-3}$ for single, double, and tripe QW lasers for this case). From Fig. 4 , it is clearly observed that the energy shift $\Delta E^{(1)}(=14 \mathrm{meV})>\Delta E^{(2)}(=9.7$ $\mathrm{meV})>\Delta E^{(3)}(=8.2 \mathrm{meV})$ at the same carrier density difference $\left(\Delta N_{\mathrm{th}}=2 \times 10^{18} \mathrm{~cm}^{-3}\right)$ for single, double, and tripe $\mathrm{QW}$ lasers, i.e., the energy shift becomes smaller at a larger number of wells.

At a further increase of the carrier density $\left(N_{\mathrm{th}} \geq 9 \times\right.$ $10^{18} \mathrm{~cm}^{-3}$, and $N_{w}=1$ ), the occurrence of the second quantized state transition is added into the value of peak gain. The lasing energy, which corresponds to the second quantized state transition, jumps to a shorter wavelength. This transition due to the second quantized state at a short cavity length QW lasers has been observed by Mittelstein et al. [31]. At the same carrier injections, the quasi-Fermi levels about double and triple QW lasers increase less rap- idly to the second quantized state than that in a single QW laser, so the second quantized state transition is not found for a larger number of wells QW laser.

\section{B. Effects of Barrier Height}

Fig. 5 demonstrates the relative position of emission energy as a function of barrier height $(x=0.2,0.4)$ at high carrier injections $\left(N_{\mathrm{th}}=1 \times 10^{18}-1 \times 10^{19} \mathrm{~cm}^{-3}\right)$, and $L_{w} / L_{b}=101 \AA / 51 \AA$.

For either a low or a high barrier QW laser, the emission energy increases to a larger energy with an increase of carrier density. As discussed in the previous part, this blue shift is enhanced by the band filling and the gain broadening effects.

As shown in Fig. 5, the emission energy increases while the barrier height is increased. This is due to a smaller transition energy about $n=1$ subband $\left(E_{n=1, \mathrm{e} \rightarrow \mathrm{hh}}\right)$ in a low barrier height $\left(x_{\mathrm{Al}}=0.2\right) \mathrm{QW}$. For a high barrier $\left(x_{\mathrm{Al}}\right.$ $=0.4) \mathrm{QW}$ structure, of which wavefunctions are more confined in the well, the transition energy is larger. These transition energies of $n=1$ for $x_{\mathrm{Al}}=0.2$ and $x_{\mathrm{Al}}=0.4$ are 1.4573 and $1.4624 \mathrm{eV}$ respectively (the carrier effect is not taken into consideration). The transition energy of $x_{\mathrm{Al}}=0.4$ is larger than that of $x_{\mathrm{Al}}=0.2$, and the difference between these two transition energies is about 5.1 $\mathrm{meV}$. Besides, the energy shift due to carrier density for $x_{\mathrm{Al}}=0.4$ is only slightly larger than the shift for $x_{\mathrm{Al}}=$ 0.2 . Fig. 5 shows that the value of $\Delta E$ is equal to 14.5 $\mathrm{meV}$ for $x_{\mathrm{Al}}=0.4$, and is slightly larger than $13.6 \mathrm{meV}$ for $x_{\mathrm{Al}}=0.2$ while $\Delta N_{\mathrm{th}}$ is equal to $2 \times 10^{18} \mathrm{~cm}^{-3}$.

When the threshold carrier density is up to $8 \times 10^{18}$ $\mathrm{cm}^{-3}$ for $\left(x_{\mathrm{Al}}=0.2\right)$ single QW lasers, the transition from lasing at $n=1-2\left(E_{n=2, \mathrm{e} \rightarrow \mathrm{hh}}\right)$ subbands occurs. However, the second quantized state transition of a high barrier $\left(x_{\mathrm{Al}}=0.4\right)$ single $\mathrm{QW}$ laser doesn't takes place till the carrier density reaches $1 \times 10^{19} \mathrm{~cm}^{-3}$, and demonstrates a larger emission energy than that of the lower barrier one. This is caused by the higher transition energy about $n=1$ subband and the larger difference of transition energy between $n=1$ and $n=2$ subbands transitions for $x_{\mathrm{Al}}=0.4$. In single $\mathrm{QW}$ structures, while the carrier effect is neglected, the transition energies due to $n=2$ subband are 1.5531 and $1.5782 \mathrm{eV}$ for $x_{\mathrm{Al}}=0.2$ and $x_{\mathrm{Al}}$ $=0.4 .\left(L_{w}=101 \AA\right)$. Therefore, the difference of the second state transition between $x_{\mathrm{Al}}=0.4$ and $x_{\mathrm{Al}}=0.2$ is about $25.1 \mathrm{meV}$. When the carrier effect is considered, this tendency remains unchanged. The quasi-Fermi energy for $x_{\mathrm{Al}}=0.2$ increases quickly to the second quantized level than that for $x_{\mathrm{Al}}=0.4$ because there's a smaller difference between $n=1$ and $n=2$ for the former. Hence the transition due to the second quantized state for $x_{\mathrm{Al}}=$ 0.2 lasers occurs at a lower threshold carrier density, and the emission energy of $x_{\mathrm{Al}}=0.2$ is smaller than that of $x_{\mathrm{Al}}=0.4$.

For a double $\mathrm{QW}$ or a larger number of wells MQW lasers. The second quantized state transitions for low or high barrier MQW lasers are not observed in this range. 


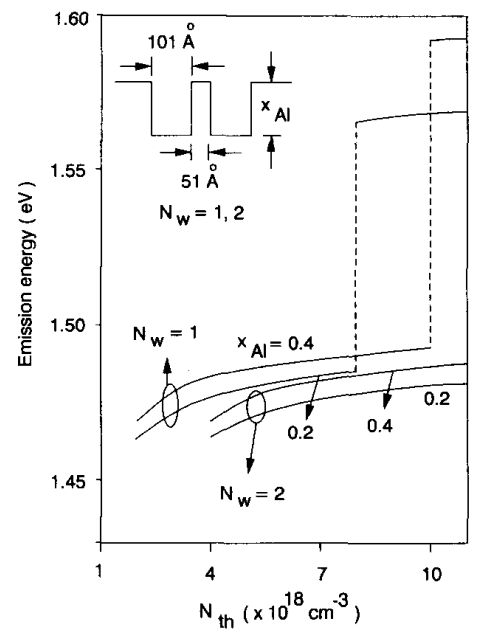

Fig. 5. Emission energy under high carrier injections $\left(N_{\mathrm{th}}=1 \times 10^{18}-1\right.$ $\left.\times 10^{19} \mathrm{~cm}^{-3}\right)$ for various barrier height $x_{\mathrm{Al}}=0.2,0.4$ and $\left(N_{w}=1,2\right)$, $L_{b}=51 \AA, L_{w}=101 \AA$ (where the bandgap shrinkage effect is not considered).

The emission energy of high $\left(x_{\mathrm{Al}}=0.4\right)$ or low $\left(x_{\mathrm{Al}}=\right.$ $0.2) \mathrm{QW}$ lasers is primarily determined by the $n=1$ subband transition. The higher barrier height has a larger emission energy than the lower barrier height.

\section{Effects of Well Width}

In designing $\mathrm{QW}$ laser diodes, the thickness of well is also one of important parameters in determining QW properties [6], [7]. Saint-Cricq et al. have proposed there is an optimum well width value which gives the lowest threshold current at a certain gain [6]. In this calculation, the well widths are chosen to be $75 \AA$ and $125 \AA$ at high carrier density $\left(N_{\text {th }}=1 \times 10^{18}-1 \times 10^{19} \mathrm{~cm}^{-3}\right)$, where barrier height and barrier width are 0.3 and $51 \AA$ respectively.

Fig. 6 shows the emission energy is increased with a decrease of well width due to a larger separation of electrons and holes levels in a thin well. That is, the thinner well width causes the gain peak to shift to a shorter wavelength. In addition, the energy shifts due to carrier density $\left(\Delta E / \Delta N_{\text {th }}\right)$ are $18.5 \mathrm{meV} / 2 \times 10^{18} \mathrm{~cm}^{-3}$ and $84 \mathrm{meV} / 2$ $\times 10^{18} \mathrm{~cm}^{-3}$ for $L_{w}=75 \AA$ and $125 \AA$ at $N_{\text {th }}=4 \times$ $10^{18} \mathrm{~cm}^{-3}$ in single QW's. This is explained that the difference of the Fermi-Dirac distribution $\left(f_{c}-f_{v}\right)$ is enhanced in thin well QW's at lower injections such that the lasing wavelength in the thick well shows a smaller shift than that in the thin well. When the threshold carrier density is above $4 \times 10^{18} \mathrm{~cm}^{-3}$, the shift for $L_{w}=75 \AA$ becomes more smooth. For double QW's, a less obvious turning point is occurred at around $7 \times 10^{18} \mathrm{~cm}^{-3}$. While the well width becomes thick $\left(L_{w}=125 \AA\right.$ ) , the turning point is not existed for either single or double $\mathrm{QW}$ lasers.

The second quantized state transition only occurs for thick $\left(L_{w}=125 \AA\right.$ ) single QW lasers at carrier density equal to $6 \times 10^{18} \mathrm{~cm}^{-3}$. For a thin well $\left(L_{w}=75 \AA\right)$,

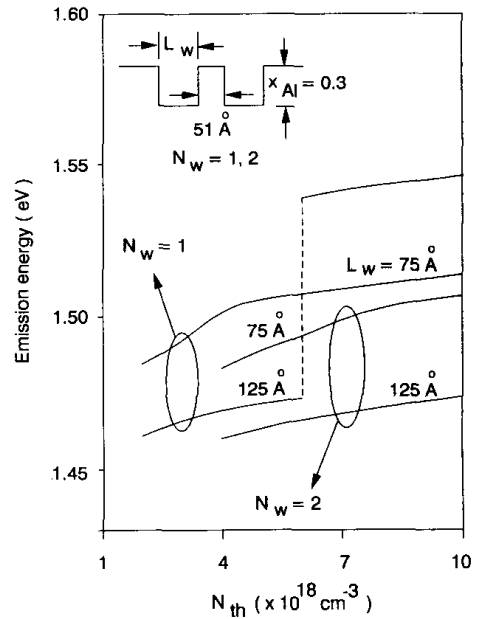

Fig. 6. Emission energy under high carrier injections $\left(N_{\mathrm{th}}=1 \times 10^{18}-1\right.$ $\times 10^{19} \mathrm{~cm}^{-3}$ ) for various well width $L_{w}=75,125 \AA$ and $N_{w^{\prime}}=1,2, L_{b}$ $=51 \AA, x_{\mathrm{Al}}=0.3$ (where the bandgap shrinkage effect is not considered).

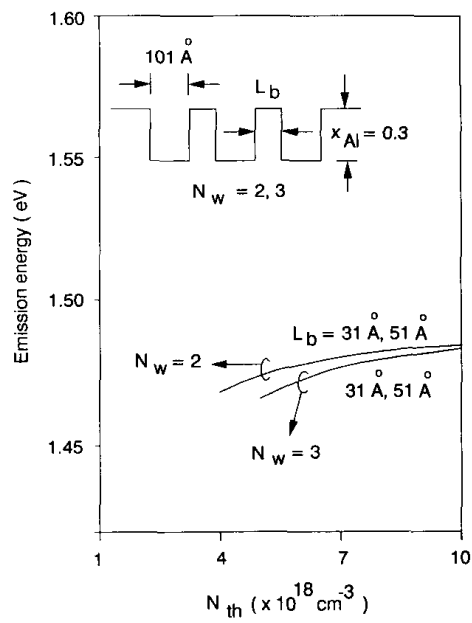

Fig. 7. Emission energy under high carrier injections $\left(N_{\mathrm{th}}=1 \times 10^{18}-1\right.$ $\times 10^{19} \mathrm{~cm}^{-3}$ ) for various barrier width $L_{b}=31,51 \AA$ and $N_{\mathrm{w}}=2,3, L_{\mathrm{w}}$ $=101 \AA, x_{\mathrm{Al}}=0.3$ (where the bandgap shrinkage effect is not considered).

the separation between $n=1$ and $n=2$ is large, so it is hard to cause transition due to the second quantized state.

\section{Effects of Barrier Width}

Fig. 7 shows the emission energies due to different barrier width $\left(L_{b}=31 \AA, 51 \AA\right)$ at high injections $\left(N_{\text {th }}=\right.$ $\left.1 \times 10^{18}-1 \times 10^{19} \mathrm{~cm}^{-3}\right)$, where well width and barrier height is equal to $101 \AA$ and 0.3 .

When the barrier is thin $\left(L_{b}=31 \AA, 51 \AA\right.$ in this case satisfy the condition), the coupling between adjacent wells makes the degeneracy of the individual well quantized energy levels disappear and each well level splits into $N_{w}$ different energy subbands. The splitting in a thinner bar$\operatorname{rier}\left(L_{b}=31 \AA\right)$ is somewhat larger than that in a thicker 
barrier $\left(L_{b}=51 \AA\right)$. At high carrier density, the quasiFermi energy difference $\left(E_{f n}-E_{f p}\right)$ is always larger than $n=1$ top subband transition energy, and lower than the energy of $n=2$ bottom subband transition. So the influence of the band filling and the gain broadening effects on a thin or a thick barrier QW lasers is almost the same. It makes no difference for the lasing wavelength by changing the barrier width.

\section{The Bandgap Shrinkage Effect in MQW LASERS}

\section{A. Comparisons of Band Structures}

Fig. 8(a) and (b) show single and double quantum well structures potential profiles and ground state wavefunctions in the conduction and the valence bands, where the threshold carrier density is $5 \times 10^{18} \mathrm{~cm}^{-3}, L_{w}=101 \AA$, $L_{b}=51 \AA$, and $x_{\mathrm{Al}}=0.3$.

When the bandgap shrinkage effect is neglected (as solid line shown), the variation of potential profiles is only influenced by the free carrier screening effect. Because the heavy holes $\left(m_{\mathrm{hh}}^{*}=0.34 m_{0}\right)$ have a larger effective mass than the electrons $\left(m_{\mathrm{e}}^{*}=0.067 m_{0}\right)$, the holes are more confined in the wells than the electrons. Fig. 8(a) shows both the conduction and valence bands bend downward for single QW's [19]. In double QW's, there's a smaller influence on the potential profiles due to the free carrier screening effect, so the bending of the potential is less obvious than that in single QW's, as shown in Fig. 8(b).

While the bandgap shrinkage effect is considered (as dashed line shown), the effective bandgap becomes narrow due to the adding of exchange-correlation interactions. The bandgap shrinkage effect reduces the bandgap with an increase of the carrier density, so the potential is lower than that without considering it at high injections. The potential profile of conduction band bends more downward, and the potential profile of valence band bends upward. However, in single QW's, the minimum point is not at the center of the well because of the influence of the screening effect which makes the valence band potential bends downward, and demonstrates a strong interaction at the well center [11]. In double QW'S, the influence of the free carrier screening is reduced, such that the valence band potential profile demonstrates flat rather than bends downward around the centers of both wells.

As the bandgap shrinkage effect are considered in the QW structures, the ground state energy levels in double QW's have a smaller shift than those in single QW's. These levels for electrons shift to lower side $\left(E_{\mathrm{e} 11}: 1.605\right.$ $\mathrm{eV} \rightarrow 1.587 \mathrm{eV}$ for a single QW, $1.608 \mathrm{eV} \rightarrow 1.593 \mathrm{eV}$ for a double $\mathrm{QW})$, and shift to upper side for holes $\left(E_{\mathrm{e} 11}\right.$ : $0.144 \mathrm{eV} \rightarrow 0.167 \mathrm{eV}$ for a single QW, $0.147 \mathrm{eV} \rightarrow 0.166$ $\mathrm{eV}$ for a double $\mathrm{QW}$ ). However, these associated ground state wavefunctions obtained by considering the bandgap shrinkage effect in the conduction band and valence band are almost unchanged to those without this effect.

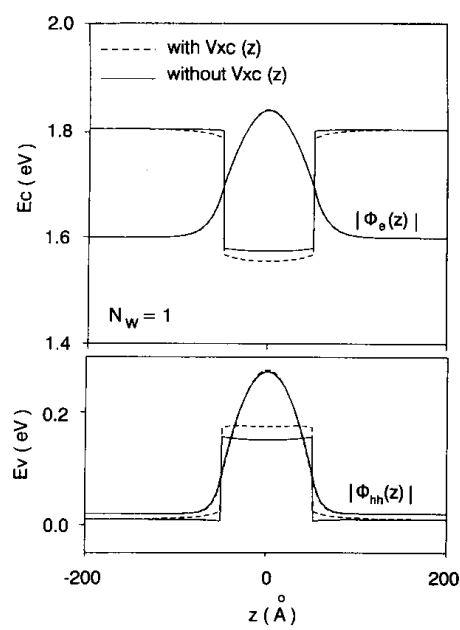

(a)

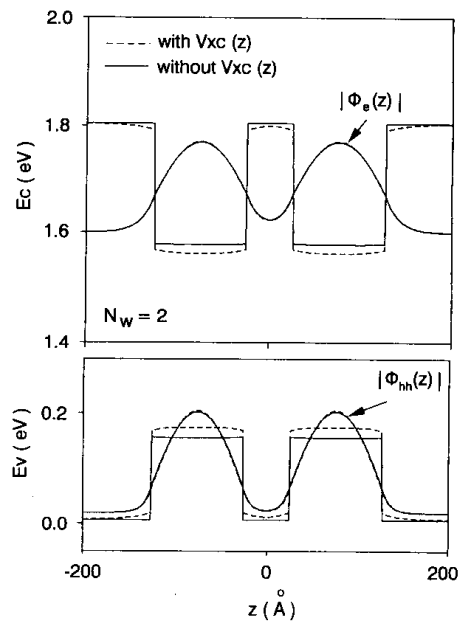

(b)

Fig. 8. Band structures and the ground state wavefunctions $\left(\Psi_{e}, \Psi_{\mathrm{bh}}\right)$ in the conduction and valence bands for (a) single $\left(N_{w}=1\right)$ and (b) double QW's $\left(N_{w}=2\right)$, where $L_{w}=101 \AA, L_{b}=51 \AA, x_{\mathrm{Al}}=0.3, N_{\mathrm{th}}=5 \times$ $10^{18} \mathrm{~cm}^{-3}$. The solid line is calculated without the bandgap shrinkage ef fect, and the dashed line is calculated with the bandgap shrinkage effect, where the bandgap shrinkage effect is represented by $V_{x c}(z)$.

\section{B. Comparisons of Emission Energy Shift}

Emission energy shifts with and without the consideration of the bandgap shrinkage effect are calculated, as Fig. 9 shown. A single and a double QW lasers are taken into consideration, where well width, barrier width, and barrier height are $101 \AA, 51 \AA$, and 0.3 . The carrier density is from $1 \times 10^{18}-1 \times 10^{19} \mathrm{~cm}^{-3}$.

While the bandgap shrinkage effect is not considered, the peak position as a function of threshold carrier density mostly depends on the band filling and the gain broadening effects. These two effects give rise to a blue shift on the lasing wavelength with an increase of the threshold carrier density. The band filling effect, which is determined by the position of the quasi-Fermi levels relative to the corresponding bandedge, shifts the gain peak toward higher energy [13], [22]. The broadening of gain 


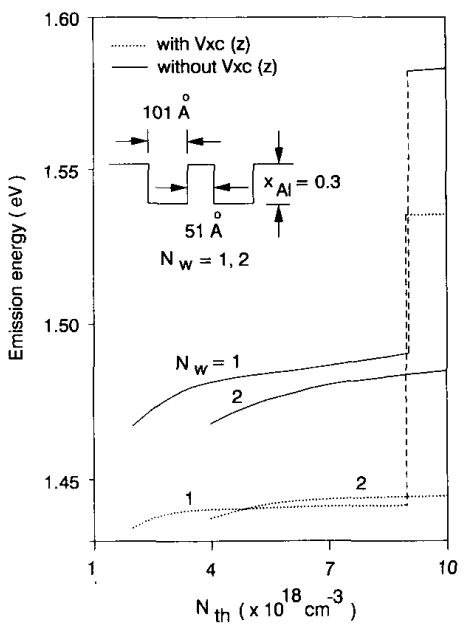

Fig. 9. Comparisons of emission energies with and without the consideration of the bandgap shrinkage effect at high carrier injections for single and double QW lasers $\left(N_{w}=1,2\right)$, where $L_{b}=51 \AA, L_{w^{\prime}}=101 \AA, x_{\mathrm{A}}$ $=0.3$, and $N_{\mathrm{th}}=1 \times 10^{18}-1 \times 10^{19} \mathrm{~cm}^{-3}$. The solid line is calculated without the bandgap shrinkage effect, and the dotted line is calculated with the bandgap shrinkage effect, where the bandgap shrinkage effect is represented by $V_{x c}(z)$.

spectrum is due to the intraband scattering which is characterized by the intraband relaxation lifetime $\tau_{\text {in }} \propto N^{-1 / 2}$ in two dimensional structures. The intraband relaxation makes a shift of gain peak toward shorter wavelength with increasing the carrier density.

When the bandgap shrinkage effect is considered, this effect may cause a rigid reduction of the bandgap at high carrier density. The bandgap shrinkage provides a red shift to a lower energy. The occurrence of bandgap narrowing balances the effects of band filling and gain broadening process on the gain peak position.

In Fig. 9, the emission energy shift increases to a higher side with an increase of the carrier density while the bandgap shrinkage is neglected. The larger number of wells has a smaller shift dependence on the carrier density, as discussed in Section III. However, when the bandgap shrinkage effect is considered, the emission energy shift for a single or a double QW lasers is almost insensitive to the carrier density. The emission energy remains fixed in the range of carrier densities between $3 \times 10^{18}$ and $9 \times$ $10^{18} \mathrm{~cm}^{-3}$ for single QW's, and above $6 \times 10^{18} \mathrm{~cm}^{-3}$ for double QW's. Moreover, the influence of the bandgap shrinkage on double QW's is smaller at higher injections, so the emission energy of double QW lasers is slightly larger than that of single QW lasers (below the second quantized state transition).

\section{Comparisons of Gain Spectra}

Fig. 10(a) and (b) show the gain spectra calculated at different carrier densities $\left(N_{\text {th }}=2,5\right.$, and $10 \times 10^{18}$ $\mathrm{cm}^{-3}$ ) for a single and a double $\mathrm{QW}$ lasers, where well width, barrier width, barrier height are $101 \AA, 51 \AA$, and 0.3

In Fig. 10(a), the gain spectra for single QW lasers are

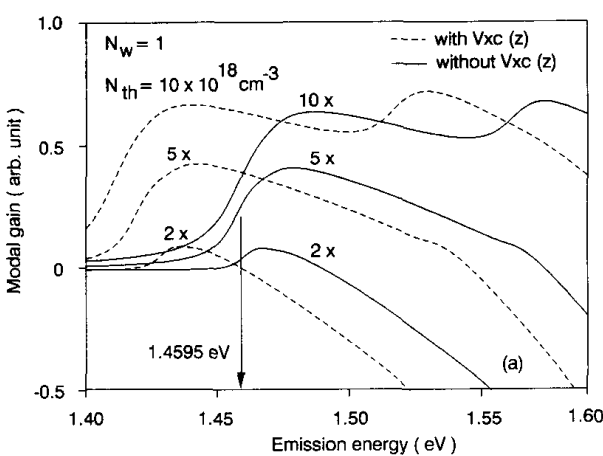

(a)

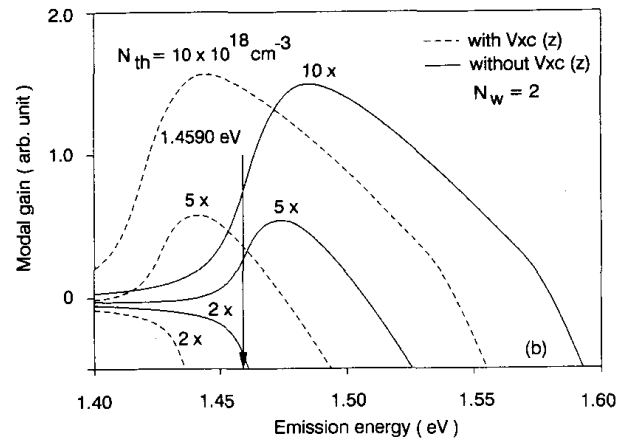

(b)

Fig. 10. Modal gain spectra for (a) single $\left(N_{w}=1\right)$ and (b) double QW lasers $\left(N_{w^{\prime}}=2\right)$, where $L_{w}=101 \AA, L_{b}=51 \AA, N_{\mathrm{th}}=2,5$, and $10 \times$ $10^{18} \mathrm{~cm}^{-3}$ respectively. The solid line is calculated without the bandgap shrinkage effect, and the dashed line is calculated with the bandgap shrinkage effect, where the bandgap shrinkage effect is represented by $V_{x c}(z)$ The position indicated by the arrow shows the transition energy due to $n$ $=1,\left(E_{\mathrm{e} \mid 1 \rightarrow, h 11}\right)$, equal to 1.4595 and $1.4590 \mathrm{eV}$ for single QW's and double $\mathrm{QW}$ 's respectively.

calculated. There are three effects to be considered. First, the gain spectra are rounded due to the presence of gain broadening. When compared to no gain broadening $\left(\tau_{\text {in }}\right.$ $\rightarrow \infty, \tau_{\text {in }}$ is no longer a carrier-dependent lifetime), there exist a blue shift on gain peak, an increase of the spectra width of the gain profile, and a reduction of the maximum gain for the former [29], [35]. Second, due to the band filling effect, the emission energy increases to higher lasing energy as the carrier density is increased. The gain spectrum shows a larger broadening at higher injections. At a further increase of carrier density, a shoulder on the high energy side is observed, indicating the occurrence of the transition of the second quantized state. Third, the bandgap shrinkage effect is taken into consideration (as dashed line shown). These gain curves shift to lower energy location, and the peak gain value is slightly larger than that without the bandgap shrinkage. The bandgap shrinkage effect causes more carriers to be confined in the well.

In Fig. 10(b), the gain spectra for double QW lasers are shown. The gain spectra are also rounded by the gain broadening effect, which is the same as that for single QW lasers. Moreover, the emission energy shifts to a higher energy side with an increase of carrier density. While the 
bandgap shrinkage is considered, the gain spectra shift to a lower lasing energy. The gain peaks become larger, and the gain profiles remains almost the same shape as that without the bandgap shrinkage effect.

When compared to single QW lasers, the double QW lasers have a larger peak gain at the same threshold carrier density. The spectrum width is narrower for double $\mathrm{QW}$ lasers, and the transition due to the second quantized state is not observed. In addition, the laser diodes with or without consideration of the bandgap shrinkage effect are not lased for the carrier density at $2 \times 10^{18} \mathrm{~cm}^{-3}$ in double QW lasers. For a larger number of wells lasers, a higher threshold carrier density is required to obtain the lasing condition.

\section{CONCLUSION}

Emission energy shift on MQW laser diodes due to high carrier density at threshold is calculated theoretically. The Schrödinger and the Poisson equations are used to solve this problem self-consistently. Furthermore, based on the Fermi's Golden Rule, the gain spectra with carrier density-dependent lifetime broadening are calculated. The emission energy shows a blue shift due to the band filling effect with gain broadening. The emission energy and the energy shift due to the band filling and the gain broadening can be adjusted by MQW device parameters. In MQW lasers, larger number of wells, lower barrier height, or wider well thickness shows a smaller energy shift dependence on the carrier density. The barrier width has little influence on the energy shift. At high injections, the occurrence of the bandgap shrinkage may manifest a rigid reduction of the bandgap, and balances the band filling and the gain broadening effects. The influence of this red shift in MQW lasers is smaller than that in single $Q W$ lasers. Besides, at a further increase of carrier density, the second quantized state transition is found in single QW's, however it is difficult to be observed in MQW's.

\section{REFERENCES}

[1] L. Esaki, "A bird's-eye view on the evolution of semiconductor superlattices and quantum wells," IEEE J. Quantum Electron., vol. QE-22, pp. 1611-1624, 1986.

[2] H. Okamoto, "Semiconductor quantum well structures for optoelectronics-Recent advances and future prospects," Jpn. J. Appl. Phys., vol. 26, pp. $315-330,1987$.

[3] H. Iwamura, T. Saku, H. Kobayashi, and Y. Horikoshi, "Spectrum studies on a GaAs-AIGaAs multi-quantum-well laser diode grown by molecular beam epitaxy," J. Appl. Phys., vol. 54, pp. 2692-2695, 1983.

[4] N. Holonyak, Jr., R. M. Kolbas, D. Dupuis, and P. D. Dapkus, "Quantum-well heterostructure lasers," IEEE J. Quantum Electron., vol. QE-16, pp. 170-186, 1980.

[5] W. T. Tsang, "Heterostructure semiconductor lasers prepared by molecular beam epitaxy," IEEE J. Quantum Electron., vol. QE-20, pp. 1119-1132, 1984

[y] B. Saint-Cricq, F. Lozes-Dupuy, and G. Vassilieff, "Well width dependence of gain and threshold current in GaAlAs single quantum well lasers," IEEE J. Quantum Electron., vol. QE-22, pp. 625-630, 1986.

[7] Y. Arakawa and A. Yariv, "Theory of gain, modulation response, and spectral linewidth in AlGaAs quantum well lasers," IEEE $J$. Quantum Electron., vol. QE-21, pp. 1666-1674, 1985.
[8] W. T. Tsang, "Extremely low threshold (AlGa)As modified multiquantum well heterostructure lasers grown by molecular-beam epitaxy," Appl. Phys. Lett., vol. 39, pp. 786-788, 1981.

[9] S. Tarucha, Y. Horikoshi, and H. Okamoto, "Optical absorption characteristics of GaAs-AlGaAs multi-quantum-well heterostructure waveguides," Jpn. J. Appl. Phys., vol. 22, pp. L482-L484, 1983.

[10] S. Tarucha, H. Kobayashi, Y. Horikoshi, and H. Okamoto, "Carrierinduced energy-gap shrinkage in current-injection GaAs/AlGaAs MQW heterostructures,"'Jpn. J. Appl. Phys., vol. 23, pp. 874-878, 1984.

[11] A. Tomita and A. Suzuki, "Carrier-induced lasing wavelength shift for quantum well laser diodes," IEEE J. Quantum Electron., vol. QE-23, pp. 1155-1159, 1987

[12] T. Ando, "Density-functional calculation of sub-band structure in accumulation and inversion layers,"' Phys. Rev., B13, pp. 3468-3477, 1976.

[13] R. Nagarajan, T. Kamiya, and A. Kurobe, "Band filling in GaAs/AIGaAs multiquantum well lasers and its effect on the threshold current," IEEE J. Quantum Electron., vol. QE-25, pp. 1161$1170,1989$.

[14] A. Kurobe, H. Furoyama, S. Naritsuka, N. Sugiyama, Y. Kokubun, and M. Nakamura, "Effects of well number, cavity length, and facet reflectivity on the reduction of threshold current of GaAs/AIGaAs multiquantum well lasers," IEEE J. Quantum Electron., vol. QE-24, pp. $635-640,1988$.

[15] E. Zielinski, H. Schweizer, S. Hausser, R. Stuber, M. H. Pilkuhn, and G. Weimann, "Systematics of laser operation in GaAs/AlGaAs multiquantum well heterostructures," IEEE J. Quantum Electron., vol. QE-23, pp. 969-976, 1987.

[16] C. Juang, K. J. Kuhn, and R. B. Darling, "Stark shift and fieldinduced tunneling in $\mathrm{Al}_{x} \mathrm{Ga}_{1-} \mathrm{As} / \mathrm{GaAs}$ quantum well structures," Phys. Rev., B41, pp. 12 047-12 053, 1990.

[17] A. Sugimura, "Threshold currents for AlGaAs quantum well lasers," IEEE J. Quantum Electron., vol. QE-20, pp. 336-343, 1984

[18] F. Stern, and S. Das. Sarma, "Electron energy levels in GaAs$\mathrm{Ga}_{1-x} \mathrm{Al}_{x} \mathrm{As}$ heterojunctions,"' Phys. Rev., B30, pp. 840-848, 1984.

[19] D. Ahn and S. L. Chuang, "Model of the field-effect quantum-well laser with free-carrier screening and valence band mixing," J. Appl. Phys., 64, pp. 6143-6149, 1988.

[20] F. Stern, "Iteration methods for calculating self-consistent field in semiconductor inversion layers,"' J. Computational Phys., vol. 6, pp. 56-67, 1970

[21] B. A. Vojak, W. D. Laidig, N. Holonyak, Jr., M. D. Camras, J. J. Coleman, and P. D. Dapkus, "High-energy (visible red) stimulated emission in GaAs," J. Appl. Phys., vol. 52, pp. 621-626, 1981.

[22] P. Blood, S. Colak, and A. I. Kucharska, "Influence of broadening and high-injection effects on GaAs-AlGaAs quantum well lasers,' IEEE J. Quantum Electron., vol. QE-24, pp. 1593-1604, 1988.

[23] G. Duggan, "A critical review of heterojunction band offsets," $J$. Vac. Sci. Tech., Technol. B, vol. 3, pp. 1224-1230, 1985.

[24] A. R. Reisinger, P. S. Zory, Jr., and R. G. Waters, "Cavity length dependence of the threshold behavior in thin quantum well semiconductor lasers," IEEE J. Quantum Electron., vol. QE-23, pp. 993$999,1987$.

[25] M. Asada, A. Kameyama, and Y. Suematsu, “Gain and intervalence band absorption in quantum well lasers," IEEE J. Quantum Electron., vol. QE-20, pp. 745-753, 1984.

[26] A. I. Kucharska and D. J. Robbins, "Lifetime broadening in GaAsAlGaAs quantum well lasers," IEEE J. Quantum Electron., vol. QE26, pp. 443-448, 1990.

[27] G. P. Agrawal and N. K. Dutta, Long Wavelength Semiconductor Lasers. New York: Van Nostrand Reinhold, 1986, ch. 9.

[28] E. O. Kane, "Band structure of indium antimonide,' J. Phys. Chem. Solids, vol. 1, pp. 249-261, 1957.

[29] M. Yamada, S. Ogita, M. Yamagishi, and K. Tabata, "Anisotropy and broadening of optical gain in a GaAs/AlGaAs multiquantumwell laser," IEEE J. Quantum Electron., vol. QE-21, pp. 640-645, 1985.

[30] M. Yamanishi and I. Suemune, "Comment on polarization dependent momentum matrix elements in quantum well lasers," Jpn. J. Appl. Phys., vol. 23, pp. L35-L36, 1984.

[31] M. Mittelstein, Y. Arakawa, A. Larrson, and A. Yariv, "Second quantized state lasing of a current pumped single quantum well laser," Appl. Phys. Lett., vol. 49, pp. 1689-1691, 1986.

[32] S. R. Chinn, P. S. Zory, and A. R. Reisinger, "A model for GRINSCH-SQW diode lasers," IEEE J. Quantum Electron., vol. QE-24, pp. 2191-2214, 1988. 
[33] P. W. A. McIlroy, A. Kurobe, and Y. Uematsu, "Analysis and application of theoretical gain curves to the design of multiquantum well lasers," IEEE J. Quantum Electron., vol. QE-21, pp. 1958-1963, 1985.

[34] P. T. Landsberg, M. S. Abrahams, and M. Osinski, "Evidence of no $k$-selection in gain spectra of quantum well AlGaAs laser diodes," IEEE J. Quantum Electron., vol. QE-21, pp. 24-28, 1985.

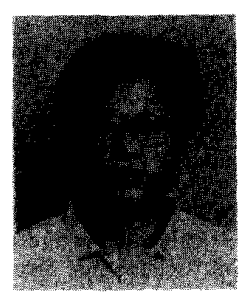

Po-An Chen ( $S^{\prime} 90$ ) was born in Chia-yi, Taiwan, in 1968 . He received the B.S. degree in electrical engineering from the National Cheng-Kung University, Tainan, Taiwan, in 1989.

Currently, he is pursuing the Ph.D. degree at the Institute of Electronics Engineering of the $\mathrm{Na}$ tional Chiao-Tung University, Hsinchu, Taiwan. His research interests are in semiconductor lasers, and high-speed quantum-well optoelectronic devices.

C. Juang, photograph and biography not available at the time of publication.

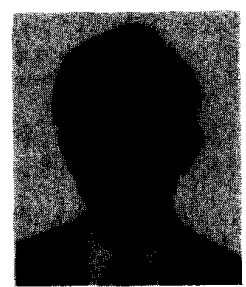

Chun-Yen Chang (S'69, M'70, SM'81, F'88) was born in Kaohsuing, Taiwan, Republic of China, on October 12, 1937. He received the B.S. degree in electrical engineering from National Chen Kung University, Tainan, Taiwan, in 1960 , and the M.S. and Ph.D. degrees from National Chiao Tung University in 1962 and 1970, respectively.

From 1962 to 1966 , he was a research assistant and then an instructor at Chiao Tung University working on organizing a semiconductor research laboratory. During 1966-1976, he was first an associate professor and later a professor in solid-state electronics and semiconductor physics and technologies; he was also chairman of the Department of Electrophysics at Chiao Tung University, and was a professor and director of the Institute of Electrical Engineering, National Chen Kung University, during 1977-1987, where he established a strong research and development base in electrical and computer engineering. In 1981, he became a member of the technical staff at Bell Laboratories, Murray Hill, NJ, working in the VLSI Device Group. He was a visiting professor at the university of Florida, Gainesville, in 1987 and the university of Stuttgart, Stuttgart, W. Germany in 1989, where he taught MBE technologies and devices. He has consulted for ERSO/ITRI, Nippon Seiki Co., UMC, etc. on VLSI, scanner, and flat panel display devices. He is currently a professor of the Institute of Electronics and Dean of the College of Engineering of National Chiao Tung University. His recent interests include silicon VLSI physics and technologies, III-V compound devices, materials using MBE and MOCVD, and amorphous-silicon devices. He has taught the majority of senior semiconductor engineers in Taiwan since 1962, and published more than 120 technical papers in international journals. He has contributed carrier transport theory and specific contact resistivity in metal-semiconductor systems $(1970,1971)$. He invented a method of MOS surface stabilization (1966), a method of low-pressure MOCVD using a TEG source (1981), amorphous Si photo-transistors (1986), the quantum-well base high-speed transistor (1986), and the bipolar-unipolar transition negative resistance transistor (BUNDR, 1987), etc.

Dr. Chang is a member of Phi Tau Phi, the American Electromagnetics Academy, the Chinese Institute of Electrical Engineers, the American Physical Society, and the Electrochemical Society. He was the recipient of an academic achievement award in engineering from the Ministry of Education and the distinguished research award of the National Science Council, Republic of China, and the recipient of the 1989-1990 international traveling award granted by the China Foundation to distinguished scholar in the Republic of China, Hou's Material Science Award 1992, R.O.C. for his contribution to low pressure MOCVD.

He has been elected an IEEE fellow for "his contribution to semiconductor devices development and to education." 